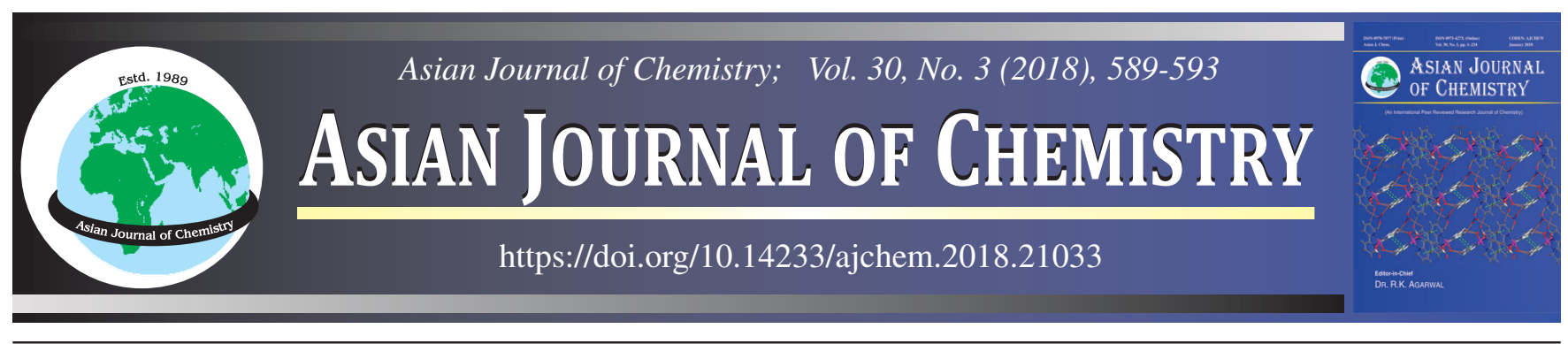

\title{
Palladium(II) Acetate as Catalyst in Transvinylation \\ Reactions of Hydroxycinnamic Acid and Its Derivatives
}

\section{L.O. KADIDAE ${ }^{1, *}$, A. UsAMI ${ }^{2}$ and M. HONDA ${ }^{2}$}

${ }^{1}$ Department of Chemistry Faculty of Mathematics and Natural Sciences, Halu Oleo University, Kendari, Southeast Sulawesi, 93232, Indonesia ${ }^{2}$ Division of Material Sciences, Graduate School of Natural Science and Technology, Kanazawa University, Kakuma-Machi, Kanazawa, 9201192, Japan

*Corresponding author: Fax: +62 4013190496; Tel: +62 85242588078; E-mail: 1kadidae@ gmail.com

Received: 7 September 2017; Accepted: 9 November 2017;

Published online: 31 January 2018;

AJC-18750

The study on application of palladium(II) acetate as catalyst in transvinylation reactions of hydroxycinnamic acids has been done. This study was intended to assess the capability of palladium(II) acetate as a safer replacement for mercuric(II) catalysts in transvinylation reactions of hydroxycinnamic acids and its derivatives. Optimizing the performance of the catalyst by applying several additional catalysts in the reactions was firstly done. Among those catalysts, $\mathrm{KOH}$ and $\mathrm{H}_{2} \mathrm{SO}_{4}$ showed significant impacts on the yields of the transvinylation reactions. It increased to $96 \%$ when $\mathrm{KOH}$ was added and to $95 \%$ when $\mathrm{H}_{2} \mathrm{SO}_{4}$ was added, compared to only $66 \%$ when palladium(II) acetate alone was employed. Adding either $\mathrm{KOH}$ or $\mathrm{H}_{2} \mathrm{SO}_{4}$ along with the primary catalyst of palladium(II) acetate in transvinylation reactions of hydroxycinnamic acids gave the desired products in good yields. This proved that palladium(II) acetate was capable of catalyzing the transvinylation reactions of hydroxycinnamic acid and its derivatives to produce some vinyl esters.

Keywords: Palladium(II) acetate, Catalyst, Hydroxycinnamic acid, Transvinylation.

ᄂ

\section{INTRODUCTION}

Efforts to find the most effective way of esterification reaction of carboxylic acids with secondary or tertiary alcohols are still interesting and challenging. Two basic approaches to improve yields in chemical syntheses of common esters of carboxylic acids with alcohols are employing an excess of alcohol to drive the balance of the reaction equation towards products and removal of water as one of the esterification products $[1,2]$.

In certain conditions, for example, when esters intended to possess bulky structures of secondary or tertiary alcohols, such as quinic acid and its derivatives, those techniques are not enough to boost yields against steric hindrances [3]. One way to cope with this matter is the use of vinyl esters as starting material to react with those bulky alcohols through a transesterification reaction. This is particularly interesting; however, the effective and safe methods of synthesizing vinyl esters remains unrevealed. Reported methods of vinyl esters syntheses were relied mostly on the use of mercuric catalysts, such as mercury(II) sulfate or mercury(II) acetate to enhance the vinyl ester products. The transvinylation reaction employing vinyl acetate alongside with mercuric salts was first introduced by Herrmann et al. [4] and Toussaint and McDowell Jr. [5]
Adelman [6] studied vinyl esterification reaction using mercuric acetate(II) based on infrared absorption spectrum. Kavitha et al. [7] reported the synthesis of vinyl caffeate with low yield. Other reports that used mercury(II) acetate as catalyst to synthesize vinyl caffeate were also reported [8-10]. Gao et al. [11] reported the synthesis of vinyl coumarate using mercury(II) acetate.

Despite being good catalysts, mercuric compounds in all oxidation states are highly toxic to the environment and human health. On the other hand, palladium is relatively eco-friendly and less harmful to human health. Palladium has been reported in numerous publications showing its versatility as organopalladium intermediates in various synthetic organic chemistry. For example, palladium(II) acetate and palladium catalyst uses were reported by Yang et al. [12] to provide vinyl esters by coupling carboxylic acids and alkenes but not a transvinylation. And in some reports the use of $\mathrm{Pd}(\mathrm{II})$ and $\mathrm{Hg}$ (II) as catalysts are inter-changeable [13] and to this fact, herein, palladium(II) acetate was proposed to be a safer replacement for $\mathrm{Hg}(\mathrm{II})$ as catalyst in transvinylation reaction of hydroxycinnamic acid. This article is reporting the results of optimization condition of palladium(II) acetate as catalyst for transvinylation especially in accordance with the use of some additional catalysts. The results of trans-vinylation reaction of hydroxycinnamic acid 
and its derivatives with vinyl acetate in the presence of palladium(II) acetate as catalyst were also presented.

\section{EXPERIMENTAL}

Palladium(II) acetate, cinnamic acid, vinyl acetate, dihydroxycinnamic acid, 4-hydroxycinnamic acid, ferulic acid, 4-tert-butyldimethylsiloxycinnamic acid, 4-tert-butyldimethylsiloxy-3-cinnamic acid, 3,4-di-tert-butyldimethoxycinnamic acid were bought from Merck, Nakalai Tesque, TCI or Kanto. Solvents used were purified using known methods. All reactions were conducted in dried glassware under argon atmosphere. Thin layer chromatographic (TLC) analyses were performed on Kieselgel $60 \mathrm{~F}_{254}$ plates from Merck. Detection was carried out under UV light or spraying with $20 \%$ ethanolic sulfuric acid. Flash chromatography for substance purifications and separations were performed on Silica Gel 60N, 40-50 $\mu \mathrm{m}$. Solvents evaporation was performed using Iwaki Rotary Evaporator REN-1000 with reduced pressure. Products that have been available in literatures, their ${ }^{1} \mathrm{H}$ NMR were analyzed and matched to references. New compound products, their ${ }^{1} \mathrm{H}$ and ${ }^{13} \mathrm{C}$ NMR spectra, IR spectra and HRMS spectra were analyzed to confirm their structures. JEOL NMR of JNMLA400 and ECA500 were utilized in analyses of ${ }^{1} \mathrm{H}$ and ${ }^{13} \mathrm{C}$ NMR spectra of the synthetic products. JEOL JMS-700 was used to record high resolution mass spectrophotometer (HRMS) spectra. HORIBA FT-720 FT-IR spectrometer was used to record infrared spectra.

Synthesis of vinyl cinnamate (2): The procedure of the syntheses of various vinyl esters was adapted from Gao et al. [11]. In a two-neck flask, cinnamic acid (1) $(1.0 \mathrm{mmol}, 148$ $\mathrm{mg})$, THF (1 mL), vinyl acetate $(16 \mathrm{mmol}, 1.4 \mathrm{~g}, 1.5 \mathrm{~mL})$ and palladium(II) acetate $(0.1 \mathrm{mmol}, 9 \mathrm{mg})$ were added together and the reaction mixture was stirred for $30 \mathrm{~min}$ at room temperature. Thereafter, $10 \% \mathrm{w} / \mathrm{w}$ of sulfuric acid in THF (1 drop) was added and the mixture was continued to stir for $4 \mathrm{~h}$ at $40{ }^{\circ} \mathrm{C}$, then filtered through celite. The filtrate was washed with water $(15 \mathrm{~mL} \times 1)$ and saturated with brine $(15 \mathrm{~mL} \times 2)$. The organic layer was dried over sodium sulfate and concentrated using an evaporator to get desired product (95 \%). ${ }^{1} \mathrm{H}$ NMR (400 MHz, $\left.\left(\mathrm{CD}_{3}\right)_{2} \mathrm{CO}, \delta, \mathrm{ppm}\right): 4.66$ (dd, $1 \mathrm{H}, J$ $=6.3,1.5 \mathrm{~Hz}, \mathrm{CH}-\mathrm{C}$-vinyl), 4.96 (dd, $1 \mathrm{H}, J=14.0,1.96 \mathrm{~Hz}$, CH-C-vinyl), 6.62 (d, 1H, J = 16.1 Hz, CO-CH-CH-Ph), 7.39$7.47(\mathrm{~m}, 4 \mathrm{H} ; 3 \mathrm{H}, \mathrm{Ar}-\mathrm{H}$ and $1 \mathrm{H}, \mathrm{O}-\mathrm{CH}$-vinyl), 7.37-7.81 (d, $2 \mathrm{H}, J=8.6 \mathrm{~Hz}, \mathrm{Ar}-\mathrm{H}), 7.83$ (d, 1H, $J=16.0 \mathrm{~Hz}, \mathrm{Ph}-\mathrm{CH}-\mathrm{CH}-$ $\mathrm{CO}$ ). Proton NMR data is in agreement with literature [14]. Each experiment in Table-1 was performed similarly but using different additives: $p$-TsOH, $\mathrm{BF}_{3} \cdot \mathrm{OEt}_{2}$, pyridine, $\mathrm{K}_{2} \mathrm{CO}_{3}, \mathrm{KOH}$.

Synthesis of vinyl caffeate (4): Dihydroxycinnamic acid (3) $(1.0 \mathrm{mmol}, 194 \mathrm{mg})$ was placed into a two-necked flask, THF ( $1 \mathrm{~mL})$, vinyl acetate $(16 \mathrm{mmol}, 1.4 \mathrm{~g}, 1.5 \mathrm{~mL})$ and palladium(II) acetate $(0.1 \mathrm{mmol}, 9 \mathrm{mg})$ were also added and the mixture was stirred for $30 \mathrm{~min}$ at room temperature. Thereafter, $10 \%$ w/w of sulfuric acid in THF (1 drop) was added and the mixture was stirred for $4 \mathrm{~h}$ at $40^{\circ} \mathrm{C}$, filtered through celite and the filtrate was concentrated using an evaporator. The crude material was purified over chromatography column $(n$-hexane: EtOAc, 1:1) to give desired product $\mathbf{4}$, as yellow solid, $\mathrm{R}_{\mathrm{f}}=$ $0.41,\left(21 \%\right.$ yield). ${ }^{1} \mathrm{H}$ NMR (500 $\left.\mathrm{MHz}, \mathrm{CD}_{3} \mathrm{OD}, \delta, \mathrm{ppm}\right)$ : $4.61(\mathrm{dd}, 1 \mathrm{H}, J=6.3,1.1 \mathrm{~Hz}, \mathrm{CH}$-C-vinyl), $4.94(\mathrm{dd}, 1 \mathrm{H}, J=$ 14.0, 1.4 Hz, CH-C-vinyl), 6.29 (d, 1H, $J=15.5 \mathrm{~Hz}$, CO-CHCH-Ph), 6.79 (d, $1 \mathrm{H}, J=8.0 \mathrm{~Hz}, \mathrm{Ar}-\mathrm{H}), 6.99$ (dd, $1 \mathrm{H}, J=8.3$, $2.0 \mathrm{~Hz}, \mathrm{Ar}-\mathrm{H}), 7.07$ (d, 1H, $J=2.3 \mathrm{~Hz}, \mathrm{Ar}-\mathrm{H}), 7.40$ (dd, $1 \mathrm{H}, J$ $=13.7,6.3 \mathrm{~Hz}, \mathrm{O}-\mathrm{CH}$-vinyl), 7.66 (d, $1 \mathrm{H}, J=16.0 \mathrm{~Hz}, \mathrm{Ph}-$ $\mathrm{CH}-\mathrm{CH}-\mathrm{CO}$ ). Data is in agreement with literature [7].

Synthesis of vinyl coumarate (6): 4-Hydroxycinnamic acid (5) $(1.0 \mathrm{mmol}, 164 \mathrm{mg})$ was placed into a two-necked flask, THF ( $1 \mathrm{~mL})$, vinyl acetate $(16 \mathrm{mmol}, 1.4 \mathrm{~g}, 1.5 \mathrm{~mL})$ and palladium(II) acetate $(0.1 \mathrm{mmol}, 9 \mathrm{mg})$ were also added and the mixture was stirred for $30 \mathrm{~min}$ at room temperature. Thereafter, $10 \% \mathrm{w} / \mathrm{w}$ of sulfuric acid in THF ( 1 drop) was added and the mixture was stirred for $4 \mathrm{~h}$ at $40{ }^{\circ} \mathrm{C}$. The mixture was filtered through celite and the filtrate was concentrated using an evaporator. The crude material was purified over chromatography column ( $n$-hexane:EtOAc, 2:1) to give desired product $\mathbf{6}$, as yellow solid, $\mathrm{R}_{\mathrm{f}}=0.35$, (35\% yield). ${ }^{1} \mathrm{H}$ NMR $(500 \mathrm{MHz}$, $\mathrm{CDCl}_{3}, \delta, \mathrm{ppm}$ ): 4.63 (dd, $1 \mathrm{H}, J=6.3,1.1 \mathrm{~Hz}, \mathrm{CH}-\mathrm{C}$-vinyl), 4.98 (dd, 1H, $J=13.7,1.7 \mathrm{~Hz}, \mathrm{CH}-\mathrm{C}$-vinyl), 5.6 (s, broad, $1 \mathrm{H}, \mathrm{OH}), 6.32$ (d, 1H, J=16.0 Hz, CO-CH-CH-Ph), 6.87 (d, $2 \mathrm{H}, J=8.6 \mathrm{~Hz}, \mathrm{Ar}-\mathrm{H}$ ), 7.42 (dd, 1H, $J=13.7,6.3 \mathrm{~Hz}, \mathrm{O}-\mathrm{CH}-$ vinyl), 7.48 (d, 2H, $J=8.6 \mathrm{~Hz}, \operatorname{Ar}-\mathrm{H}), 7.74$ (d, 1H, $J=16.0$ $\mathrm{Hz}, \mathrm{Ph}-\mathrm{CH}-\mathrm{CH}-\mathrm{CO})$. Data is in agreement with literature [11].

Synthesis of vinyl ferulate (8): Ferulic acid (7) (1.0 mmol, $194 \mathrm{mg}$ ) was placed into a two-necked flask, THF (1 mL), vinyl acetate $(16 \mathrm{mmol}, 1.4 \mathrm{~g}, 1.5 \mathrm{~mL})$ and palladium(II) acetate $(0.1 \mathrm{mmol}, 9 \mathrm{mg})$ were also added and the mixture was stirred for $30 \mathrm{~min}$ at room temperature. Thereafter, $10 \% \mathrm{w} / \mathrm{w}$ of sulfuric acid in THF (1 drop) was added and the mixture was stirred for $4 \mathrm{~h}$ at $40{ }^{\circ} \mathrm{C}$. The mixture was filtered through celite and the filtrate was concentrated using an evaporator. The crude material was purified over chromatography column ( $n$-hexane: EtOAc, 2:1) to give desired product 8 , as yellow solid, $\mathrm{R}_{\mathrm{f}}=$ $0.42,\left(16 \%\right.$ yield). ${ }^{1} \mathrm{H}$ NMR (500 MHz, $\left.\mathrm{CDCl}_{3}, \delta, \mathrm{ppm}\right): 3.93$ (s, $3 \mathrm{H}, \mathrm{CH}_{3}$ ), 4.62 (d, $1 \mathrm{H}, J=6.3 \mathrm{~Hz}, \mathrm{CH}-\mathrm{C}$-vinyl), 4.96 (d, $1 \mathrm{H}, J=13.7 \mathrm{~Hz}, \mathrm{CH}-\mathrm{C}$-vinyl), 5.98 (s, broad, $1 \mathrm{H}, \mathrm{OH}), 6.31$ $(\mathrm{d}, 1 \mathrm{H}, J=16.0 \mathrm{~Hz}, \mathrm{CO}-\mathrm{CH}-\mathrm{CH}-\mathrm{Ph}), 6.92(\mathrm{~d}, 1 \mathrm{H}, J=8.0 \mathrm{~Hz}$, Ar-H), 7.09 (d, 2H, $J=8.0 \mathrm{~Hz}, \mathrm{Ar}-\mathrm{H}), 7.43(1 \mathrm{H}, \mathrm{dd}, J=13.7$, $6.3 \mathrm{~Hz}, \mathrm{O}-\mathrm{CH}$-vinyl), 7.72 (d, 1H, $J=16.0 \mathrm{~Hz}, \mathrm{Ph}-\mathrm{CH}-\mathrm{CH}-$ $\mathrm{CO})$. Data is in agreement with literature [11].

Synthesis of vinyl 4-tert-butyldimethylsiloxycinnamate (10): 4-tert-Butyldimethylsiloxycinnamic acid (9) $(1.0 \mathrm{mmol}$, $278 \mathrm{mg}$ ) was placed into a two-necked flask. To this, vinyl acetate $(16 \mathrm{mmol}, 1.4 \mathrm{~g}, 1.5 \mathrm{~mL})$ and palladium(II) acetate $(0.1 \mathrm{mmol}$, $9 \mathrm{mg}$ ) were also added and the reaction mixture was stirred for $30 \mathrm{~min}$ at room temperature. Then, $\mathrm{KOH}(0.1 \mathrm{mmol}, 6 \mathrm{mg})$ was added and the reaction mixture was stirred for $4 \mathrm{~h}$ at $40{ }^{\circ} \mathrm{C}$. The mixture was filtered through celite and the filtrate was concentrated using evaporator and purified with column chromatography over silica to give $70 \%$ the desired product 10. $\left(n\right.$-hexane:EtOAc, $\left.30: 1, \mathrm{v} / \mathrm{v}, \mathrm{R}_{\mathrm{f}}=0.40\right)$. IR (film, $\left.v_{\max }, \mathrm{cm}^{-1}\right)$ : 3087 (C-H, alkene), 3033 (C-H, aromatic), 2955 (C-H, methyl), 1731 (C=O, carbonyl), 1509 (C=C, aromatic), 1149 (C-O, ester). ${ }^{1} \mathrm{H} \mathrm{NMR}\left(500 \mathrm{MHz}, \mathrm{CDCl}_{3}, \delta, \mathrm{ppm}\right): 0.23\left(\mathrm{~s}, 6 \mathrm{H},\left(\mathrm{CH}_{3}\right)_{2}-\mathrm{Si}\right)$ ), 0.99 (s, 9H, $\left.\left(\mathrm{CH}_{3}\right)_{3} \mathrm{C}\right), 4.62$ (dd, $1 \mathrm{H}, J=6.3,1.5 \mathrm{~Hz}, \mathrm{CH}-\mathrm{C}-$ vinyl), 4.97 (dd, $1 \mathrm{H}, J=14.0,1.6 \mathrm{~Hz}, \mathrm{CH}-\mathrm{C}$-vinyl), 6.32 (d, $1 \mathrm{H}, J=16.1 \mathrm{~Hz}, \mathrm{CO}-\mathrm{CH}-\mathrm{CH}-\mathrm{Ph}$ ), 6.86 (t, $2 \mathrm{H}, J=4.3 \mathrm{~Hz}$, ArH), 7.40-7.46 (m, 2H, Ar-H; 1H, CH-vinyl), 7.74 (d, 1H, $J=$ 
$16.1 \mathrm{~Hz}, \mathrm{Ph}-\mathrm{CH}-\mathrm{CH}-\mathrm{CO}) .{ }^{13} \mathrm{CNMR}\left(125 \mathrm{MHz}, \mathrm{CDCl}_{3}, \delta, \mathrm{ppm}\right)$ : 164.3 (1C, carboxylic-C), 158.4 (1C, Ar-C), 146.5 (1C, Ph-C), 141.5 (1C, Ar-C), 130.1 (1C, CH-Vin), 127.5 (2C, Ar-C), 120.7 (2C, Ar-C), 114.3 (1C, benz-C), 97.5 (1C, $\mathrm{CH}_{2}$-Vin), 25.7 (3C, $\left.\left(\mathrm{CH}_{3}\right)-\mathrm{C}\right), 18.3\left(1 \mathrm{C}, \mathrm{C}-\mathrm{CH}_{3}\right),-4.3\left(2 \mathrm{C},\left(\mathrm{CH}_{3}\right)_{2}-\mathrm{Si}\right)$. HRMS (EI): $m / z$ : calcd. for $\mathrm{C}_{17} \mathrm{H}_{24} \mathrm{O}_{3} \mathrm{Si}[\mathrm{M}]^{+}, 304.1495$; found 304.1488.

Synthesis of vinyl 4-tert-butyldimethylsiloxy-3-methoxycinnamate (12): 4-tert-Butyldimethylsiloxy-3-cinnamic acid (11) $(1.0 \mathrm{mmol}, 317 \mathrm{mg})$ was placed into a two-necked flask. To this vinyl acetate $(16 \mathrm{mmol}, 1.4 \mathrm{~g}, 1.5 \mathrm{~mL}$ ) and palladium(II) acetate $(0.1 \mathrm{mmol}, 9 \mathrm{mg})$ were also added and the reaction mixture was stirred for $30 \mathrm{~min}$ at room temperature. Then, $\mathrm{KOH}(0.1 \mathrm{mmol}, 6 \mathrm{mg})$ was added and the reaction mixture was stirred for $4 \mathrm{~h}$ at $40{ }^{\circ} \mathrm{C}$. The mixture was filtered through celite and the filtrate was concentrated using evaporator and purified with column chromatography over silica to give $50 \%$ the desired product 12. ( $n$-hexane:EtOAc, 30:1, $\mathrm{R}_{\mathrm{f}}=0.40$ ). IR (film, $\left.v_{\max }, \mathrm{cm}^{-1}\right): 3086(\mathrm{C}-\mathrm{H}$, alkene), 3047 (C-H, aromatic), 2929 (C-H, methyl), 1727 (C=O, carbonyl), 1511 ( $\mathrm{C}=\mathrm{C}$, aromatic), 1146 (C-O, ester), 1036 ( $\mathrm{C}=\mathrm{CH}_{2}$, vinyl). ${ }^{1} \mathrm{H}$ NMR $(500 \mathrm{MHz}$, $\left.\left.\mathrm{CDCl}_{3}, \delta, \mathrm{ppm}\right): 0.12\left(\mathrm{~s}, 6 \mathrm{H},\left(\mathrm{CH}_{3}\right)_{2}-\mathrm{Si}\right)\right), 0.94\left(\mathrm{~s}, 9 \mathrm{H},\left(\mathrm{CH}_{3}\right)_{3} \mathrm{C}\right)$, 3.78 (s, $3 \mathrm{H}, \mathrm{CH}_{3}-\mathrm{O}$ ), 4.56 (dd, $1 \mathrm{H}, J=6.3,1.7 \mathrm{~Hz}, \mathrm{CH}-\mathrm{C}$-vinyl), 4.90 (dd, $1 \mathrm{H}, J=14.0,1.4 \mathrm{~Hz}, \mathrm{CH}-\mathrm{C}$-vinyl), 6.26 (d, 1H, $J=$ $15.5 \mathrm{~Hz}, \mathrm{CO}-\mathrm{CH}-\mathrm{CH}-\mathrm{Ph}), 6.80(\mathrm{~d}, 1 \mathrm{H}, J=8.0 \mathrm{~Hz}), 6.99$ (t, $2 \mathrm{H}, J=4.9 \mathrm{~Hz}), 7.37(1 \mathrm{H}, \mathrm{dd}, J=14.3,6.3 \mathrm{~Hz}), 7.67(\mathrm{~d}, 1 \mathrm{H}$, $J=16.0 \mathrm{~Hz}, \mathrm{Ph}-\mathrm{CH}-\mathrm{CH}-\mathrm{CO}) .{ }^{13} \mathrm{C}$ NMR $\left(125 \mathrm{MHz}, \mathrm{CDCl}_{3}, \delta\right.$, ppm): 164.3 (1C, carboxylic-C), 151.3 (1C, Ar-C), 148.1 (1C, Ph-CH), 146.9 (1C, Ar-C), 141.5 (1C, CH-Vin), 128.1 (1C, Ar-C), 122.7 (1C, Ar-C), 121.2 (1C, Ar-C), 114.3 (1C, Ar-C), 111.0 (1C, benz-CH), 97.5 (1C, $\mathrm{CH}_{2}$-Vin), $55.5\left(1 \mathrm{C}, \mathrm{CH}_{3}-\mathrm{O}\right)$, $25.7\left(3 \mathrm{C},\left(\mathrm{CH}_{3}\right)-\mathrm{C}\right), 18.6\left(1 \mathrm{C}, \mathrm{C}-\mathrm{CH}_{3}\right),-4.5\left(2 \mathrm{C},\left(\mathrm{CH}_{3}\right)_{2}-\mathrm{Si}\right)$. HRMS (EI): $\mathrm{m} / \mathrm{z}$ : calcd. for $\mathrm{C}_{18} \mathrm{H}_{26} \mathrm{O}_{4} \mathrm{Si}[\mathrm{M}]^{+}, 334.1600$; found 334.1605.

Synthesis of vinyl 3,4-di-tert-butyldimethylsiloxycinnamate (14): To a two-neck flask, 3,4-di-tert-butyldimethylsiloxycinnamic acid (13) $(613 \mathrm{mg}, 1.5 \mathrm{mmol})$, vinyl acetate $(2.3 \mathrm{~mL}$, $24 \mathrm{mmol})$ and palladium(II) acetate $(18 \mathrm{mg}, 0.15 \mathrm{mmol})$ were added. The reaction mixture was stirred at room temperature for $10 \mathrm{~min}$ and subsequently, $\mathrm{KOH}(10 \mathrm{mg}, 0.15 \mathrm{mmol})$ was added. The reaction mixture was stirred at $40^{\circ} \mathrm{C}$ for $4 \mathrm{~h}$, then diluted with $20 \mathrm{~mL}$ of EtOAc, filtered through celite and concentrated by evaporator under reduced pressure. Purification was done over column chromatography on silica gel ( $n$-hexane: EtOAc, 5:1, v/v) to give $580 \mathrm{mg}$ of compound 14, as yellow liquid. $\left(\mathrm{R}_{\mathrm{f}}=0.82\right.$ on $n$-hexane:EtOAc, $4: 1$, v/v). Yield: $81 \%$. IR (film, $\left.v_{\max }, \mathrm{cm}^{-1}\right)$ : 3005 (C-H, alkene), 2958 (C-H, methyl), 1713 ( $\mathrm{C}=\mathrm{O}$, ester), $1558(\mathrm{C}=\mathrm{C}$, aromatic), 1508 ( $\mathrm{C}=\mathrm{C}$, aromatic), $1269\left(\mathrm{Si}_{-} \mathrm{CH}_{3}\right), 1152$ (C-O, ester), $1092(\mathrm{Si}-\mathrm{O}), 915\left(\mathrm{C}=\mathrm{CH}_{2}\right.$, Vinyl). ${ }^{1} \mathrm{H}$ NMR $\left(500 \mathrm{MHz}, \mathrm{CDCl}_{3}, \delta, \mathrm{ppm}\right): 0.22(\mathrm{~s}, 6 \mathrm{H}$, $\left.\left(\mathrm{CH}_{3}\right)_{2}-\mathrm{Si}\right), 0.23$ (s, 6H, $\left.\left(\mathrm{CH}_{3}\right)_{2}-\mathrm{Si}\right), 0.99\left(\mathrm{~s}, 9 \mathrm{H},\left(\mathrm{CH}_{3}\right)_{3} \mathrm{C}\right), 1.00$ (s, 9H, $\left.\left(\mathrm{CH}_{3}\right)_{3} \mathrm{C}\right), 4.62$ (dd, $1 \mathrm{H}, J=6.6,1.4 \mathrm{~Hz}, \mathrm{CH}-\mathrm{C}$-vinyl), 4.97 (dd, $1 \mathrm{H}, J=14.0,1.4 \mathrm{~Hz}, \mathrm{CH}-\mathrm{C}$-vinyl), $(6.25$ (d, $1 \mathrm{H}, J=$ $16.0 \mathrm{~Hz}, \mathrm{CO}-\mathrm{CH}-\mathrm{CH}-\mathrm{Ph}$ ), 6.83 (d, $1 \mathrm{H}, J=9.2 \mathrm{~Hz}, \mathrm{Ar}-\mathrm{H})$, 7.03-7.05 (m, 2H, Ar-H), 7.42 (dd, 1H, $J=13.7,6.3 \mathrm{~Hz}, \mathrm{O}-\mathrm{CH}-$ vinyl), 7.67 (d, $1 \mathrm{H}, J=16.0 \mathrm{~Hz}$, Ph-CH-CH-CO). ${ }^{13} \mathrm{C}$ NMR (125 MHz, $\mathrm{CDCl}_{3}, \delta$, ppm): 164.41 (1C, Carboxylic-C), 150.08 (1C, Ar-C), 147.40 (1C, Ph-CH), 146.81 (1C, Ar-C), 141.54 (1C, CH-Vin), 127.84 (1C, Ar-C), 122.78 (1C, Ar-C), 121.32
(1C, Ar-C), 120.70 (1C, Ar-C), 114.22 (1C, benz-CH), 97.63 (1C, $\mathrm{CH}_{2}$-Vin), 26.04 (3C, $\left.\left(\mathrm{CH}_{3}\right)-\mathrm{C}\right), 26.00\left(3 \mathrm{C},\left(\mathrm{CH}_{3}\right)-\mathrm{C}\right)$, $18.65\left(1 \mathrm{C}, \mathrm{C}-\mathrm{CH}_{3}\right), 18.60\left(1 \mathrm{C}, \mathrm{C}-\mathrm{CH}_{3}\right),-3.91\left(2 \mathrm{C},\left(\mathrm{CH}_{3}\right)_{2}-\mathrm{Si}\right)$, $-3.95\left(2 \mathrm{C},\left(\mathrm{CH}_{3}\right)_{2}-\mathrm{Si}\right)$. HRMS (EI): $\mathrm{m} / z$ : calcd. for $\mathrm{C}_{23} \mathrm{H}_{38} \mathrm{O}_{4} \mathrm{Si}_{2}$ $[\mathrm{M}]^{+}, 434.2309$; found 434.2306.

Synthesis of vinyl 2,2-dimethylbenzo[ $[d][1,3]$ dioxolcinnamate (16): In a two-necked flask, compound 15 (1.0 $\mathrm{mmol}, 220 \mathrm{mg}$ ), vinyl acetate (16 mmol, $1.4 \mathrm{~g}, 1.5 \mathrm{~mL})$ and palladium(II) acetate $(0.1 \mathrm{mmol}, 9 \mathrm{mg})$ were added. After stirring for $10 \mathrm{~min}$ at room temperature, potassium hydroxide $(0.1 \mathrm{mmol}, 6 \mathrm{mg})$ was added and the mixture was stirred for $4 \mathrm{~h}$ at $40{ }^{\circ} \mathrm{C}$. The reaction mixture was filtered through celite and the filtrate was concentrated by an evaporator and purified by column chromatography to give compound $\mathbf{1 6}$ as a pale yellow oil $(84 \%)$. TLC ( $n$-hexane: EtOAc, $4: 1 \mathrm{v} / \mathrm{v}): \mathrm{R}_{\mathrm{f}}=0.64$. IR (film, $\left.v_{\max }, \mathrm{cm}^{-1}\right): 3087$ (C-H, alkene), 2992 (C-H, methyl), $1727(\mathrm{C}=\mathrm{O}$, carboxyl, $1496(\mathrm{C}=\mathrm{C}$, aromatic), $951(\mathrm{C}-\mathrm{H}$, vinyl), 809 (C-H, vinyl terminal). ${ }^{1} \mathrm{H} \mathrm{NMR}\left(500 \mathrm{MHz}, \mathrm{CDCl}_{3}, \delta\right.$, ppm): 1.69 (s, $\left.6 \mathrm{H},\left(\mathrm{CH}_{3}\right)_{2}-\mathrm{C}\right), 4.61$ (dd, $1 \mathrm{H}, J=6.3,1.7 \mathrm{~Hz}, \mathrm{CH}-\mathrm{C}-$ vinyl), 4.96 (dd, $1 \mathrm{H}, J=13.7,1.7 \mathrm{~Hz}, \mathrm{CH}-\mathrm{C}$-vinyl), 6.25 (d, $1 \mathrm{H}, J=15.5 \mathrm{~Hz}, \mathrm{CO}-\mathrm{CH}-\mathrm{CH}-\mathrm{Ph}), 6.71-7.01(\mathrm{~m}, 3 \mathrm{H}, \mathrm{Ar}-\mathrm{H})$, 7.42 (dd, 1H, $J=13.7,6.3 \mathrm{~Hz}$, O-CH-vinyl), 7.69 (d, 1H, $J=$ $15.5 \mathrm{~Hz}, \mathrm{Ph}-\mathrm{CH}-\mathrm{CO}) .{ }^{13} \mathrm{C}$ NMR ${ }^{13} \mathrm{C}$ NMR $\left(125 \mathrm{MHz}, \mathrm{CDCl}_{3}\right.$, $\delta$, ppm): 164.4 (1C, $\mathrm{C}=\mathrm{O}$, carboxyl), 150.1 (1C, Ar-C), 148.4 (1C, Phenyl-C), 146.8 (1C, Ar-C), 141.5 (1C, CH-vin), 128.1 (1C, C- $\left.\left(\mathrm{CH}_{3}\right)_{2} \mathrm{O}_{2}\right), 124.8$ (1C, Ar-C), 119.2 (1C, Ar-C), 113.9 (1C, Ar-C), 108.6 (1C, Ar-C), 106.5 (1C, Benz-C), 97.6 (1C, $\mathrm{CH}_{2}$-Vin), 26.0 (2C, $\mathrm{CH}_{3}-\mathrm{C}$ ). HRMS (EI): $\mathrm{m} / z$ : calcd. for $\mathrm{C}_{14} \mathrm{H}_{14} \mathrm{O}_{4}$ $[\mathrm{M}]^{+}, 246.0892$; found, 246.0899 .

Synthesis of vinyl 3,4-diacetoxycinnamate (18): In a two-necked flask compound 17 ( $1 \mathrm{mmol}, 290 \mathrm{mg})$, THF (1 mL), vinyl acetate $(16 \mathrm{mmol}, 1.4 \mathrm{~g}, 1.5 \mathrm{~mL})$ and palladium(II) acetate $(0.1 \mathrm{mmol}, 9 \mathrm{mg})$ were added. The reaction mixture was stirred for $30 \mathrm{~min}$ at room temperature. Thereafter, $10 \% \mathrm{w} / \mathrm{w}$ of sulfuric acid in THF (1 drop) was added and the mixture was stirred for $4 \mathrm{~h}$ at $40{ }^{\circ} \mathrm{C}$. The mixture was filtered through celite and the filtrate was extracted with ethyl acetate $(15 \mathrm{~mL} \times 3)$ then washed with saturated brine $(15 \mathrm{~mL} \times 2)$. The organic layer was dried over sodium sulfate and concentrated using an evaporator. Purification was done by column chromatography to give compound 18 as pale yellow oil (84\%). TLC ( $n$-hexane: EtOAc, 3: $1 \mathrm{v} / \mathrm{v}$ ): $\mathrm{R}_{\mathrm{f}}=0.30$. IR (film, $\left.v_{\max }, \mathrm{cm}^{-1}\right): 3090(\mathrm{C}-\mathrm{H}$, alkene), 2932 (C-H, methyl), 1722 (C=O, carbonyl), 1506 ( $\mathrm{C}=\mathrm{C}$, aromatic), 1167 (C-O, ester), $903\left(\mathrm{C}=\mathrm{CH}_{2}\right.$, vinyl). ${ }^{1} \mathrm{H}$ NMR (500 MHz, $\mathrm{CDCl}_{3}, \delta$, ppm): 2.31 (s, $\left.3 \mathrm{H}, \mathrm{CH}_{3}\right), 2.32$ (s, $3 \mathrm{H}, \mathrm{CH}_{3}$ ), $4.65(\mathrm{dd}, 1 \mathrm{H}, J=6.3,1.7 \mathrm{~Hz}, \mathrm{CH}-\mathrm{C}-\mathrm{vinyl}), 4.98(\mathrm{dd}, 1 \mathrm{H}, J=$ $13.7,1.7 \mathrm{~Hz}, \mathrm{CH}-\mathrm{C}$-vinyl), 6.40 (d, $1 \mathrm{H}, J=16.0 \mathrm{~Hz}, \mathrm{CO}-\mathrm{CH}-$ $\mathrm{CH}-\mathrm{Ph}$ ), 7.24 (d, 1H, $J=8.6 \mathrm{~Hz}, \mathrm{O}-\mathrm{CH}$-vinyl), 7.38-7.45 (m, $3 \mathrm{H}$, aromatic-H), $7.73(\mathrm{~d}, 1 \mathrm{H}, J=16.0 \mathrm{~Hz}, \mathrm{Ph}-\mathrm{CH}-\mathrm{CO}) .{ }^{13} \mathrm{CNMR}$ (125 MHz, $\mathrm{CDCl}_{3}, \delta, \mathrm{ppm}$ ): 168.0 (1C, C=O, acetyl), 167.9 (1C, $\mathrm{C}=\mathrm{O}$, acetyl), 163.4 (1C, C=O, ester), 144.6 (1C, Ar-C), 143.8 (1C, phenyl-C), 142.5 (1C, Ar-C), 141.2 (1C, CH-vin), 132.8 (1C, Ar-C), 126.6 (1C, Ar-C), 124.0 (1C, Ar-C), 122.9 (1C, Ar-C), 117.7 (1C, benz-C), 97.9 (1C, $\mathrm{CH}_{2}$-Vin), 20.6 (1C, $\left.\mathrm{CH}_{3}\right), 20.5\left(1 \mathrm{C}, \mathrm{CH}_{3}\right)$. HRMS (EI): $m / z$ : calcd. for $\mathrm{C}_{15} \mathrm{H}_{14} \mathrm{O}_{6}$ $[\mathrm{M}]^{+}:$290.0790; found: 290.0786 .

Synthesis of vinyl 3,4-dibenzyloxycinnamate (20): In a two-necked flask, compound 19 (1.0 mmol, $360 \mathrm{mg})$, vinyl 
acetate $(16 \mathrm{mmol}, 1.4 \mathrm{~g}, 1.5 \mathrm{~mL})$ and palladium(II) acetate $(0.1 \mathrm{mmol}, 9 \mathrm{mg})$ were added. After stirring for $10 \mathrm{~min}$ at room temperature, potassium hydroxide $(0.1 \mathrm{mmol}, 6 \mathrm{mg})$ was added and the mixture was stirred for $4 \mathrm{~h}$ at $40^{\circ} \mathrm{C}$. The reaction mixture was filtered through celite. The filtrate was concentrated by an evaporator and purified by column chromatography to give compound 20 as pale yellow waxy solid (64\%). TLC ( $n$ hexane:EtOAc, 5: $1 \mathrm{v} / \mathrm{v}): \mathrm{R}_{\mathrm{f}}=0.58 .{ }^{1} \mathrm{H}$ NMR $(500 \mathrm{MHz}$, ( $\mathrm{CDCl}_{3}, \delta$, ppm): 4.61 (dd, $1 \mathrm{H}, J=6.3,1.7 \mathrm{~Hz}, \mathrm{CH}$-C-vinyl), 4.95 (dd, $1 \mathrm{H}, J=14.0,1.4 \mathrm{~Hz}, \mathrm{CH}-\mathrm{C}$-vinyl), 5.17 (s, 2H, benzyl-H), 5.19 (s, 2H, Benzyl-H), 6.24 (d, 1H, $J=16.0 \mathrm{~Hz}$, CO-CH-CH-Ph), 6.91 (d, 1H, J = 8.6 Hz, Ar-H), 7.08 (dd, 1H, $J=8.3,2.0 \mathrm{~Hz}, \mathrm{O}-\mathrm{CH}$-vinyl), $7.13(\mathrm{~d}, 1 \mathrm{H}, J=2.3 \mathrm{~Hz}, \mathrm{Ar}-\mathrm{H})$, 7.31-7.46 (m, $11 \mathrm{H}, \mathrm{Ar}-\mathrm{H}), 7.67$ (d, $1 \mathrm{H}, J=15.5 \mathrm{~Hz}, \mathrm{Ph}-\mathrm{CH}-$ $\mathrm{CO}$ ). Data is in good agreement with literature [7].

\section{RESULTS AND DISCUSSION}

Effect of additives: Cinnamic acid (1) and vinyl acetate underwent acyl rearrangement in the presence of palladium(II) acetate as catalyst. The influence of various additives on obtained yields in synthesizing vinyl ester of cinnamic acid (2) was investigated. The kind of additives used in this reaction was determined according to Mastihubová's procedure [10].

As shown in Table-1, it is obvious that certain additives can improve the reaction yields. Sulfuric acid, potassium carbonate and potassium hydroxide (entries 4, 6, 7) had significant impact on increasing yield of compound 2 . On the other hand, $p$ - TsOH, $\mathrm{BF}_{3} \cdot \mathrm{OEt}_{2}$ or pyridine when added (entries 2, 3, 5) was apparently no effect, showing similar yields when reaction carried out without additive. While addition of pyridine, the reaction did not proceed.

Synthesis of vinyl esters: The reactions were conducted in mild temperature, at $40^{\circ} \mathrm{C}$ using tetrahydrofuran (THF) as

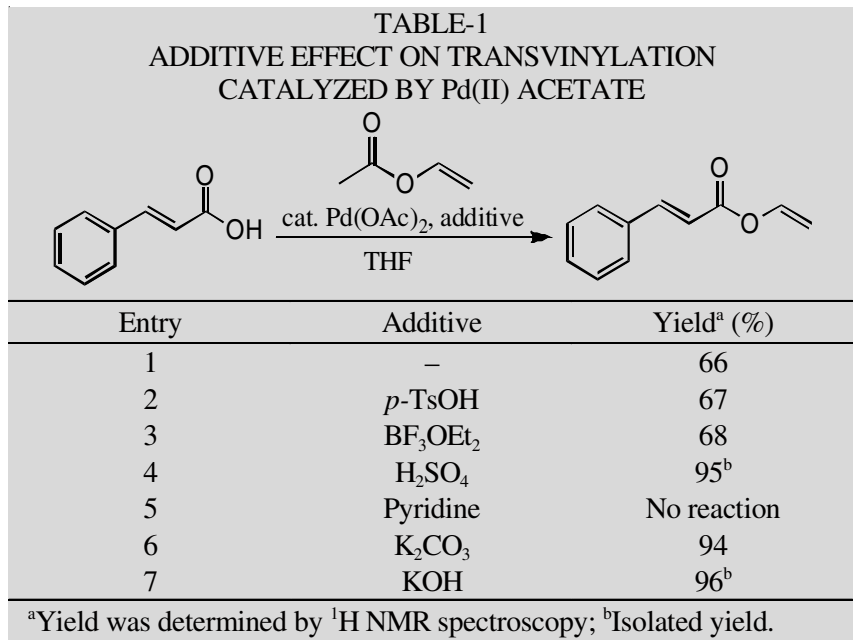

solvent for $4 \mathrm{~h}$. From the mentioned results, $\mathrm{H}_{2} \mathrm{SO}_{4}$ and $\mathrm{KOH}$ were the additives that performed better than other tested additives when cinnamic acid was reacted with vinyl caffeate. Therefore, these additives were employed in the transvinylation reaction of cinnamic acid derivatives. Dihydroxycinnamic acid (3) reacted with vinyl acetate in the presence of palladium(II) acetate and sulfuric acid as additive in THF to achieve vinyl caffeate was first investigated giving the isolated yield in $21 \%$. Coumaric acid and ferulic acid were also reacted with vinyl acetate in a similar fashion to caffeic acid giving the yields of 35 and $16 \%$, respectively. The unprotected hydroxyl groups (entries 1 to 3) had lower yields compared to those protected ones (entries 4 to 9) (Table-2). This may be related to the vulnerability of hydroxyl groups on oxidation to produce side products; hence it decreased the yields.

When the protection group was electron withdrawing group, such as acetyl (OAc) group (entry 8), the product was

TRANSVINYLATION OF HYDROXYCINNAMIC ACID AND ITS DERIVATIVES

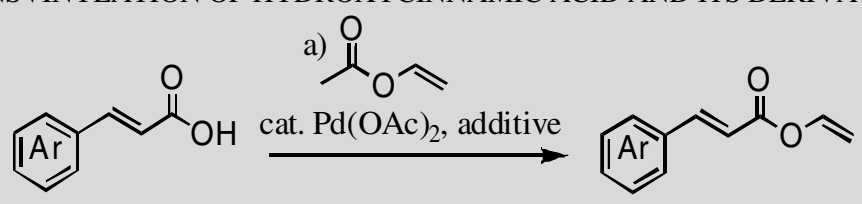

Entry


5<smiles>COc1cc(/C=C/C(=O)O)ccc1OC(C)(C)C</smiles>

11

6<smiles>CCCCOc1ccc(/C=C/C(=O)O)cc1OCC(C)(C)C</smiles>

13

7<smiles>CC1(C)Oc2ccc(/C=C/C(=O)O)cc2O1</smiles>

15

8<smiles>CC(=O)Oc1ccc(/C=C/C(=O)O)cc1OC(C)=O</smiles>

17

9
$\mathrm{KOH}$

$\mathrm{KOH}$

$\mathrm{KOH}$

$\mathrm{H}_{2} \mathrm{SO}_{4}$

$\mathrm{KOH}$<smiles>C=COC(=O)/C=C/c1ccc(OC)c(OC)c1</smiles>

12<smiles>C=COC(=O)/C=C/c1ccc(OC(C)(C)C)c(OC(C)(C)C)c1</smiles>

14<smiles>C=COC(=O)/C=C/c1ccc2c(c1)OC(C)(C)O2</smiles>

16<smiles>C=COC(=O)/C=C/c1ccc(OC(C)=O)c(OC(C)=O)c1</smiles>

18<smiles>C=COC(=O)/C=C/c1ccc(OCc2ccccc2)c(OCc2ccccc2)c1</smiles>

20
50

84

84

64

19

Reagents and conditions; a) vinyl acetate (16 equiv.), $\mathrm{Pd}(\mathrm{OAc})_{2}\left(0.1\right.$ equiv.), additive ( 0.1 equiv.), THF, $40{ }^{\circ} \mathrm{C}, 4 \mathrm{~h}$.

obtained in high yield ( $84 \%)$. On the other hand, when the electron donating groups, such as benzyl $(\mathrm{Bn})$ group protection (entry 9), obtained yield was moderate. Based on these results, the protection of hydroxyl groups of hydroxycinnamic acid was affecting the yields of transvinylation.

Two additives, $\mathrm{H}_{2} \mathrm{SO}_{4}$ and $\mathrm{KOH}$ were used differently in reactions. It was related to the stability of protecting groups. For example, tert-butyldimethylsilyl (TBS) protecting group is stable in mild basic condition but labile in mild acidic condition [15]. Acetals for diol protecting group is stable in base and susceptible with mild acid condition, while acetyl is more stable in acidic than in basic condition, that is why to cleave this protecting groups usually mild basic condition was used. Methyl ether and benzyl ether protecting group are stable in both acidic and basic condition [15].

\section{Conclusion}

Palladium(II) acetate is capable of catalyzing the syntheses of vinyl esters of cinnamic acid and its derivatives and it is able to replace the functionality of mercuric(II) catalysts. The performance of catalyst improved with the presence of additives, such as $\mathrm{H}_{2} \mathrm{SO}_{4}$ and $\mathrm{KOH}$. Obtained yields are low in the transvinylation reactions of hydroxycinnamic acids, but it turned to be significant higher when hydroxyl groups were protected prior to the transvinylation reactions.

\section{ACKNOWLEDGEMENTS}

This work has been carried out at Laboratory of Molecular Design, Kanazawa University, Kanazawa, Japan and was supported by Kanazawa University SAKIGAKE Project and Japan Society for the Promotion of Science (JSPS) KAKENHI Grant Number 15K05585.

\section{REFERENCES}

1. R.J. Fessenden and J.S. Fessenden, Organic Chemistry, Part 2, Translated in Indonesian by A.H. Pudjaatmaka, Penerbit Erlangga, Jakarta, edn 3, pp. 82-84 (1990).

2. J. McMurry and E. Simanek, Fundamentals of Organic Chemistry, Thomson Higher Education, California, edn 6, pp. 307-308 (2007).

3. L.O. Kadidae, A. Usami, T. Koyama, M. Honda and K. Kunimoto, Eur. J. Chem., 6, 367 (2015);

https://doi.org/10.5155/eurjchem.6.4.367-373.1298.

4. W.O. Herrmann and W. Haehnel, Process of Preparing Vinyl Esters, US Patent 2245131 (1941).

5. W.J. Toussaint and G. McDowell Jr., Preparation of Vinyl Esters, US Patent 2299862 (1942).

6. R.L. Adelman, J. Org. Chem., 14, 1057 (1949); https://doi.org/10.1021/jo01158a015.

7. J. Kavitha, D. Rajasekhar, G.V. Subbaraju and G.N. Ramesh, Indian J. Chem., 38B, 1280 (1999).

8. D. Swern and E.F. Jordan Jr., Org. Synth., 30, 106 (1950); https://doi.org/10.15227/orgsyn.030.0106.

9. Z. Tan and F. Shahidi, Food Chem., 133, 1427 (2012); https://doi.org/10.1016/j.foodchem.2012.02.030.

10. M. Mastihubová and V. Mastihuba, Bioorg. Med. Chem. Lett., 23, 5389 (2013); https://doi.org/10.1016/j.bmcl.2013.07.051.

11. C. Gao, P. Mayon, D.A. MacManus and E.N. Vulfson, Biotechnol. Bioeng., 71, 235 (2000); https://doi.org/10.1002/1097-0290(2000)71:3<235::AID-BIT1013> 3.0.CO;2-M.

12. D. Yang, S. Ding, J. Huang and K. Zhao, Chem. Commun., 49, 1211 (2013); https://doi.org/10.1039/c2cc37779a.

13. P.A. Caruana and A.J. Frontier, Tetrahedron, 63, 10646 (2007); https://doi.org/10.1016/j.tet.2007.08.008.

14. http://sdbs.db.aist.go.jp/sdbs/cgi-bin/direct frame top.cgi.

15. P.J. Kocienski, Protecting Groups, Stuttgart, edn 3, pp. 115-116 (2005). 